\title{
EL DISCURSO MÉDICO COMO ESTIGMA Y REFUGIO: CONCEPCIONES DE LA OBESIDAD Y SUS CONSECUENCIAS SUBJETIVAS
}

\author{
MARÍA VALENTINA NOBLIA \\ Universidad de Buenos Aires
}

\section{RESUMEN}

En la Argentina, los problemas de salud vinculados con la alimentación y la nutrición constituyen problemas sociales complejos que abarcan desde las muertes por desnutrición en un país productor de alimentos, a los cuadros de bulimia y anorexia en jóvenes de clase media, así como el incremento de casos de diabetes y de cardiopatías derivadas del crecimiento incesante de la obesidad, entre otros. Los problemas señalados se relacionan con realidades urgentes, aunque social y económicamente diferentes, a las que el Estado debe dar respuesta.

La obesidad se ha transformado en un tema central ya que el exceso de peso ha dejado de ser la señal saludable de bienestar físico y económico para constituirse en una enfermedad, a la que hay que combatir por todos los medios. Desde el Estado se han llevado a cabo diferentes programas para controlarla y prevenirla, a los que se suman las acciones de sectores privados, entre ellos, los centros médicos especializados. Estos proponen distintos enfoques médicos, con sus supuestos teóricos y abordajes terapéuticos, que son fundamentales, en la medida en que son performativos, tanto del control de la enfermedad, como de la subjetividad, las prácticas y estilos de vida del paciente.

En el marco de este trabajo analizaremos los discursos de los grupos de apoyo a las terapias de la obesidad de pacientes en tratamiento. Abordaremos las interacciones en grupos, gestionados por los propios participantes a través de las redes móviles, que ponen en escena el peso determinante de los supuestos médicos sobre sus identidades, conductas y actitudes de las personas.

El marco teórico es el del Análisis Crítico del Discurso (Fairclough, 1995, 2003, 2006), desde una perspectiva multimodal (Kress, 2010) y la metodología es cualitativa (Guba y Lincoln, 1994).

Palabras CLAVE: discurso médico, obesidad, redes sociales móviles, análisis crítico del discurso.

\section{AbSTRACT}

In Argentina, food and nutrition diseases are complex social problems that cover a wide range, from malnutrition deaths in a food producer country, to 
bulimia and anorexia in middle-class youth, among other issues like the increase in cases of diabetes and heart disease linked to the non-stopping growth of obesity. These problems mentioned are related to urgent situations, that even though rises from different social and economic contexts, the State should provide an answer.

Obesity has become a central issue for the public agenda since excess weight has ceased to be the healthy signal of physical and economic well-being to become a disease, which must be fought by all means. Different programs have been carried out by the State to control and prevent it, with the collaboration of private sectors, including specialized medical centers. They propose different medical approaches, with their theoretical assumptions and therapeutic approaches, which are fundamental, because they are performative, both on disease control, as to the subjectivity, practices and lifestyles of the patient.

In this paper we will analyze the discourse of the support groups for the therapies of obesity of patients in undergoing treatment. We will address the interactions in groups, managed by the participants themselves through the mobile networks, which set the determining weight of the medical assumptions about their identities, behaviors and attitudes.

Critical Discourse Analysis (Fairclough, 1995, 2003, 2006), from a multimodal perspective (Kress, 2010) and qualitative (Guba and Lincoln, 1994) is the theoretical and methodological framework of this paper.

KEYWORDS: medical discourse, obesity, mobile social networks, critical discourse analysis.

\section{INTRODUCCIÓN}

En la Argentina, los problemas de salud vinculados con la alimentación y la nutrición son parte de la agenda cotidiana de los medios. Las notas de diarios, programas de televisión y redes sociales traen a la escena problemas sociales complejos como las muertes por desnutrición en un país productor de alimentos; los déficits nutricionales en amplios sectores rurales estigmatizados por una pobreza ya endógena; la grave situación de chicos y adolescentes de zonas urbanas a los que la crisis económica no sólo les quita perspectivas de vida sino el alimento diario; la paradoja de ricos flacos y pobres gordos, entre otros temas. A estos tristes tópicos, se le suma otro orden de problemas alimentarios, como la bulimia y la anorexia en jóvenes de clase media, el incremento de casos de diabetes y de cardiopatías vinculadas con el crecimiento incesante de la obesidad. Los problemas señalados se relacionan con realidades urgentes, aunque social y económicamente diferentes, a las que el Estado debe dar respuesta.

En los medios, los problemas alimentarios por desnutrición y pobreza son tema de noticias esporádicas, vinculadas, fundamentalmente, con la agenda política (periodos eleccionarios, por ejemplo). Aunque los núme- 
ros de las encuestas indiquen que el problema es mucho más generalizado $^{1}$, para la prensa parece estar geo y sociolocalizado, relacionado con la realidad socioeconómica de algunas provincias y en particular con las comunidades indígenas, reforzando, así, el prejuicio hacia sectores de la población que son percibidos como ajenos a la realidad social del resto del país. En contraste, los problemas nutricionales asociados a la obesidad tienen una presencia constante a través de entrevistas a profesionales reconocidos, notas que promueven el consumo de un alimento o lo estigmatizan, dietas y ejercicios físicos, historias de vida, etc. ${ }^{2}$. El exceso de peso ha dejado de ser la señal saludable de bienestar físico y económico para constituirse en una enfermedad, a la que hay que combatir por todos los medios.

Desde el Estado se han llevado a cabo diferentes programas para controlar y prevenir los trastornos alimentarios. A poco tiempo de asumir el actual gobierno, ya se dictó la Resolución 732, en junio de 2016, y se formalizó la creación del Programa Nacional de Alimentación Saludable y Prevención de la Obesidad, poniendo en evidencia la importancia política del tema. Esta resolución vino a ampliar el alcance de la Ley 26.396, dictada en septiembre de 2008, por medio de la cual se declaraba "de interés nacional la prevención y control de los trastornos alimentarios", entendiendo por ellos a "la obesidad, a la bulimia y a la anorexia nerviosa" y demás enfermedades relacionadas. El panorama planteado por esta resolución pone en escena un estado de situación preocupante: "más de la mitad de la población argentina $(53,4 \%)$ tiene exceso de peso" y "se encuentra en claro aumento, tanto en adultos como en etapas más precoces de la vida”. Definida como una enfermedad crónica, según la Organización Mundial de la Salud, como una epidemia metabólica que afecta a la población en general, la prevención de la obesidad, entonces, es un tema central para las políticas públicas, los medios y la sociedad en general.

En el abordaje de este problema social, se suman las acciones de sectores privados. Entre ellos, los centros médicos especializados, que se han constituido en actores sociales clave para el tratamiento de esta enfermedad. Estas instituciones proponen distintos enfoques médicos, con sus supuestos teóricos y abordajes terapéuticos, que son fundamentales, en la medida en que son performativos, tanto del control de la enfermedad, como de la subjetividad, prácticas y estilo de vida del paciente. Ellos sustentan el tratamiento, definen la patología, reidentifican a las personas que

1 Según el informe de septiembre de 2016 del Instituto Nacional de Estadísticas y Censos, la pobreza en nuestro país alcanza un 32,2\% y la indigencia un $6,3 \%$.

${ }^{2}$ Los medios de comunicación, por otro lado, dedican buena parte de sus páginas al mundo gourmet, publicitando alimentos y bebidas, destinados a un público aspiracional, marcado por el devenir de la moda. 
la padecen y pautan sus relaciones y acciones, dentro y fuera del ámbito médico. Las categorías que dan cuerpo a esas representaciones sociales de la obesidad y de los obesos surgen del discurso institucional y se introducen en las distintas instancias de la vida privada de quienes padecen este trastorno. Son constitutivas de los grupos terapéuticos coordinados por profesionales, pero también de los grupos de apoyo formados exclusivamente por pacientes que buscan ayuda y contención. Decimos que son performativas, ya que, al ser reproducidas de manera dogmática y acrítica por los pacientes, transforman sus identidades, pasando de ser personas que "tienen sobrepeso" a "ser adictos a la comida", y, como tales, actúan, se identifican y se proyectan.

En el marco de este trabajo analizaremos los discursos de los grupos de apoyo a las terapias de la obesidad, desde la perspectiva de los propios pacientes en tratamiento. Las interacciones en estos grupos, gestionados por los propios participantes, ponen en escena el peso determinante de los supuestos médicos sobre sus identidades, conductas y actitudes de las personas. Analizar las categorías (Sacks, 1992) que articulan las representaciones médicas de la obesidad y cómo son reproducidas y asumidas por los mismos pacientes en sus interacciones cotidianas nos permite comprender el modo en que operan los discursos institucionalizados en las identidades de las personas con sobrepeso y obesidad. En ese sentido, proponemos como hipótesis general que la representación médica de la obesidad, entendida como adicción, cruza las diferentes prácticas sociales de los pacientes en tratamiento, produciendo cambios fundamentales en su identidad social y sus prácticas, y en sus perspectivas de vida a largo plazo.

\section{LAS REDES SOCIALES MÓVILES - LOS GRUPOS DE WHATSAPP}

La efectividad de la terapia de grupos en los tratamientos de la obesidad ha sido demostrada; constituyen una de las estrategias que definen los enfoques actuales. Tal como plantea Cormillot (2008), la base de la Pirámide Terapéutica de la Obesidad está conformada por la dieta, la ayuda profesional y los grupos terapéuticos.

Los grupos que se activan con los tratamientos son de diversos tipos: existen los grupos terapéuticos -coordinados por un profesional-, de autoayuda -conformados por pares-, de entrenamiento físico, familiares, etc. Los pacientes participan, generalmente, de más de un grupo. Este cruce de experiencias terapéuticas constituye verdaderas redes sociales presenciales y virtuales. Los grupos terapéuticos pueden definirse por ser:

[...] grupos centrados en la terapia psicológica. Su objeto principal es la curación de distintas formas de malestar psíquico [...] Forma parte de 
un proceso terapéutico y se desarrolla bajo la dirección de un profesional (o un equipo de profesionales especializados). Se procura promover la toma de conciencia de los procesos psíquicos individuales subyacentes, así como de aspectos vinculares que inciden en (y se encuentran entretejidos con) estos procesos, estimulando así cambios que tienden a restablecer o fortalecer la salud mental (Jalif, 2010).

Permiten un trabajo personalizado sobre las personas, quienes aportan su perspectiva, su historia personal, su situación particular. En estos grupos, hay una selección previa que realiza el profesional para ver la adecuación de la persona al grupo y viceversa. A diferencia de estos, los grupos de autoayuda son

organizaciones de personas que comparten similares problemas psicológicos, físicos o existenciales. En muchos casos, dichos problemas han sido estigmatizados por la sociedad más amplia y los grupos son iniciados por personas que invitan a otras a encontrarse en forma periódica y frecuente para discutir su problema común y buscar formas de resolverlo o de manejarlo de la mejor manera posible (Jalif, 2010).

La particularidad de estos grupos está en el vínculo social que sus miembros establecen; en las relaciones y el apoyo social que construyen para consolidar una red social que les permita afrontar los problemas que no puede resolver por sí solos (Jalif, 2010). Tradicionalmente estos grupos eran presenciales, se reunían en el club, la iglesia, etc., pero con el avance de Internet, los blogs, foros o chats pasaron a ser verdaderos espacios de encuentro para estos fines terapéuticos. Como señala Stommel (2009):

Con el constante aumento de la disponibilidad de Internet, desde 1993, las personas con problemas de alimentación encontraron un lugar virtual donde compartir y discutir sus preocupaciones abiertamente. [...] Estos foros ofrecen una alternativa especial para el aislamiento de las personas, especialmente de niñas, que luchan con el peso y la comida, y colabora en la busca de ayuda profesional.

A partir de 2000, con la aparición de los teléfonos inteligentes, se sumaron las redes sociales móviles (Noblia, 2015). Los grupos de apoyo a través de whatsapp permitieron la conectividad social en cualquier instancia de la vida cotidiana, incluso en el proceso mismo de movilidad. Este aspecto resultó ser funcional para el control y monitoreo de los grupos de apoyo: implicó una progresión en las posibilidades de uso en circunstancias espaciales y temporales muy diversas de la vida diaria. La zonificación (Goffman, 1974) que planteaba en sus orígenes la comunicación mediada por TICs en términos de espacio (la casa, la oficina o los cibercafés) y de tiempo (los momentos libres, la noche), se amplió al posibilitar el flujo 
de la comunicación aun en aquellos intersticios del día en los que los otros no eran posibles (Noblia, 2015). Las implicancias interpersonales y sociales de una comunicación que puede darse en "cualquier tiempo"/“cualquier lugar" fueron muchas para el apoyo grupal, en particular, por el supuesto compartido de una presencia y respuesta constante del otro. La conexión a través de mensajes breves pero frecuentes a lo largo del día intensificó la cohesión social de estos grupos, por el alto grado de inmersión que implican y por cubrir las brechas entre los encuentros cara a cara.

La articulación transmedial de las aplicaciones móviles también posibilitó formas mixtas de comunicación, combinando oralidad, escritura y demás posibilidades audiovisuales, que dieron respuesta a las restricciones de los contextos particulares (y siempre mutables) de sus usuarios (Noblia, 2015). En general, estas opciones se definen a partir de tres criterios:

a) las condiciones materiales de realización del mensaje (la posibilidad o no de escribir o hablar);

b) las ventajas comunicativas de elegir un medio u otro ${ }^{3}$;

c) la expresividad del mensaje. Estos recursos son explotados por los grupos de apoyo.

En la interacción en whatsapp, que no requiere aperturas ni cierres, el diálogo se plantea en una continuidad que sólo presenta, a lo largo del tiempo, diferencias en términos de tópicos. La presencia constante del grupo garantiza la coherencia de la conversación.

Estos grupos en línea constituyen auténticas comunidades de prácticas (Wenger, McDermott y Snyder, 2002), en el sentido de que son grupos "de personas que comparten una preocupación, un conjunto de problemas o un interés común acerca de un tema, y que profundizan su conocimiento y pericia en esta área a través de una interacción continuada”. Estas comunidades (Wenger, 1998) se sostienen por el compromiso de sus integrantes, los objetivos y el repertorio de expresiones y rutinas compartidas. En ellas, la identidad de cada uno de sus miembros es un aspecto relevante, en la medida en que su ingreso al grupo supone la asunción del problema de la obesidad y un primer paso para el proceso de curación. El supuesto sobre la efectividad de los grupos de apoyo en línea se relaciona con la

${ }^{3}$ Por ejemplo, si es necesario desarrollar un argumento o narrar un suceso, escribir implica un esfuerzo mayor de tipeo, a la vez que la oralidad permite modular a través de la prosodia el discurso, recursos que colaboran con la puesta en foco o la evaluación de un aspecto del contenido. Si, por otro lado, lo que se plantea es una transacción simple de pregunta/respuesta o la transmisión de información que conviene que quede registrada, la escritura resulta el modo más eficaz. 
modificación de aspectos de la identidad "malos" por otros más saludables (Stommel, 2009). La participación en estas comunidades tiene como objetivo un cambio en las facetas de la identidad y de las prácticas que sostienen la adicción a la comida.

\section{MARCO TEÓRICO Y METODOLOGÍA}

La metodología de este trabajo es cualitativa (Guba y Lincoln, 1994). Los casos analizados nos permiten comprender los modos en los que se construyen socialmente las relaciones interpersonales, las identidades y los roles en la interacción concreta. La perspectiva del análisis es crítica (Fairclough, 2003), en el sentido en que concebimos a los textos en el marco de las prácticas sociales en las que se realizan, entendiendo que los significados se construyen en el marco de la interacción social, como procesos dinámicos y contextualmente situados, que involucran la negociación de sus participantes. El enfoque del análisis de los textos es multimodal (Kress, 2010) en la medida en que las tecnologías habilitan nuevos modos de articular simultáneamente diferentes recursos retóricos: verbales -orales y escritos-, audiovisuales y gráficos. Para el análisis lingüístico tomamos los conceptos de la Lingüística Sistémico-Funcional (Halliday, 2004) y la Teoría de la Valoración (Martin y White, 2005), ya que nos permiten relevar en el discurso el modo en que se gestionan las relaciones interpersonales. Por último, desde una perspectiva interaccional (Goffman, 1974 y 1981) analizaremos los marcos de interpretación y el posicionamiento de los participantes en la producción del discurso.

\subsection{Corpus}

Los textos analizados provienen de un corpora mayor sobre interacción en grupos a través de tecnologías móviles (whatsapp). En él, distinguimos grupos definidos por interacciones de tipo interpersonal, sin propósitos específicos, y aquellos en los que la interacción está guiada por algún objetivo particular. Los primeros se caracterizan por la expresión de sentimientos, el intercambio de anécdotas, juegos, chistes, siempre en el marco de relaciones marcadas por la cercanía y el afecto. Los segundos se plantean como comunidades organizadas alrededor de una práctica o actividad que las convoca y que puede ser permanente o con una finalidad predefinida (Noblia, 2015).

El corpus de este trabajo está conformado por 18.926 intervenciones multimodales (orales, escritas y audiovisuales) de un grupo de whatsapp 
vinculado con un centro de salud ${ }^{4}$ que es uno de los referentes en la Argentina en el tratamiento de la obesidad. La fecha que abarca esta muestra va de febrero de 2015 a junio de 2016. Las integrantes del grupo son nueve mujeres ${ }^{5}$ entre 40 y 60 años, menos una de ellas ("Mili") que tiene veintitrés. Todas son profesionales y asisten cotidianamente a grupos terapéuticos del centro médico para mantenerse en peso.

El objetivo del Centro al que asisten es lograr un cambio en los hábitos alimentarios para que sean saludables y sustentables en el tiempo. El tratamiento consiste en una dieta básica y sesiones de terapia de grupo diarias, de dos horas de duración, coordinadas por profesionales. El grupo de whatsapp objeto de análisis fue armado por las pacientes para acompañarse y controlarse mutuamente; está regulado exclusivamente por ellas, quienes fueron fijando las pautas de comportamiento y negociando el control del grupo. No hubo intervención directa de la autoridad médica en la interacción.

Con el fin de dar cuenta de las particularidades del tema y del grupo, se sumó una muestra contrastiva de dos casos de grupos terapéuticos presenciales, coordinados por un profesional médico, dos grupos de apoyo en Facebook ${ }^{6}$ y, como marco de referencia de la voz institucional, los textos de divulgación científica publicados en el sitio web del centro médico y en la prensa.

\section{El ANÁLISIS: LA CONSTRUCCIÓN INTERACTIVA DE LA IDENTIDAD}

Los grupos de autoayuda cumplen una función intersubjetiva importante, en la medida en que están motivados por la búsqueda de contención y ayuda entre pares, fuera del circuito médico. El acompañamiento, el compromiso compartido y el afecto son los sustentos de una relación que se sostiene a través de la conversación diaria. Esa meta es estructurante del intercambio que analizaremos y que perdurará en la medida en que los objetivos se mantengan. En los textos analizados, no hay tema ni situación personal que logre desviar la conducta de las participantes ${ }^{7}$; si surgen,

${ }^{4}$ Por una cuestión ética y de confidencialidad no se hace público el nombre de la institución médica de la que se recabaron los datos. No es intención de este trabajo evaluar una práctica médica ni la pertinencia o no de un tratamiento. Los objetivos de este trabajo son discursivos.

${ }^{5}$ Por el mismo motivo, se han modificado los nombres originales de los integrantes del grupo de apoyo, quienes aparecen mencionadas con un pseudónimo.

${ }^{6}$ Los grupos terapéuticos de este Centro son de acceso público en la red, lo mismo que las páginas web y las de Facebook.

${ }_{7}$ Este aspecto los distingue claramente de otros grupos de whatsapp. 
son sólo momentáneos y son reorientados rápidamente hacia el tópico central del cuidado del peso y el mantenimiento de la conducta. Por ejemplo, ante un suceso inesperado y triste -la muerte repentina del hermano de una de las integrantes- la respuesta es empática pero orientada a los propósitos del grupo ("Isa, gracias por contarnos lo que pasó...te quiero mucho y te mando un abrazo fuerte fuerte, tratá de transitar el dolor por lo que pasó, sin pensar en la comida ni en el peso, no te enojes con vos ahora, no pierdas el camino ${ }^{\prime 8}$ ). El tópico de la comida, el del mantenimiento y sus rutinas operan globalmente, garantizando la coherencia discursiva a lo largo del tiempo.

¿Cómo se habla de estos temas en el grupo? Todas las integrantes tienen una experiencia en común, un rito de pasaje exitoso y estimulante: la dieta de descenso, que implicó cumplir la dieta y asimilar los conceptos, actitudes y prácticas requeridas por el tratamiento. El nuevo estado es el resultado de un aprendizaje, de transformar una experiencia y una identidad personal y social, a partir del reencuadramiento que le dio el saber médico. En el pasaje por la institución médica, la obesidad dejó de ser un problema de índole físico, para transformarse en un problema conductual, psicológico: la adicción a comer:

El comer es adictivo y provoca una enfermedad: la obesidad. Los alimentos, sobre todo las grasas y los hidratos de carbono refinados -azúcar y harina-, producen un efecto de necesidad porque actúan sobre los neurotransmisores cerebrales. Se la ha considerado incluso un mal cerebral, a partir de que se ha ido estudiando el circuito que permite que la comida actúe como una droga $a^{9}$.

El párrafo citado condensa el eje conceptual de la representación médica más pregnante en el grupo: la obesidad como adicción, pero no vinculada con una sustancia particular (como el alcohol o la droga), sino como una conducta relacionada con la acción humana más básica y necesaria: comer. Ese desplazamiento desde el objeto a la acción sitúa a esta adicción en un in media res entre las adicciones a las sustancias y las adicciones a prácticas nocivas como jugar o robar. Por ello, la acción central de la terapia no pasa por la abstinencia, sino por una restricción de la conducta. Como señala Jalif (2010), la comida -a diferencia de otras drogas- no permite nunca la abstinencia total, ya que siempre hay que ingerir algo para alimentarse. Por eso, el tratamiento médico se propone tratar ambos frentes, operando sobre la dimensión material externa, la comida (dosificando las

\footnotetext{
${ }^{8}$ Las cursivas son mías. A lo largo de este trabajo usaremos este recurso para destacar los recursos analizados.

${ }^{9}$ Entrevista realizada al Director del Centro Médico en el diario La Nación (13-4-2006).
} 
porciones como si fueran "vacunas"), y sobre su dimensión interna, la conductual, localizada en el cerebro, entendido tanto como el lugar en el que se procesan las sustancias adictivas, como dimensión psicológica:

[...] comer en pequeñas cantidades. Este es nuestro método. No dar grasas, sino provocar la salida de la grasa. Esta grasa que se elimina es la que provoca la saciedad. Y la poca cantidad de comida es la que va a hacer que se mueva la grasa depositada. Ahora bien, esta poca comida es una dieta variada; de la comida que engorda, la pequeña porción es la "vacuna". Nuestro método está basado en corte, medida y distancia. El corte inmediato con los excesos; la medida en la porción, el cuerpo y la ropa, y la distancia entre las comidas y con la comida ${ }^{10}$.

En esta definición, que proviene de la autoridad médica, la nominalización, típica de los discursos científicos, invisibiliza al sujeto, poniendo el foco en los procesos y en los objetos: es "el comer" lo que es adictivo y provoca la obesidad; es "la comida" la que actúa como droga. En ese mismo sentido, el cuerpo aparece fragmentado, definido por sus partes ("grasa", "cuerpo", "cerebro", "ropa”), objetivizado como recipiente, como depósito que puede llenarse o vaciarse según la "cabeza" o conducta de la persona.

El control de esta parte -la cabeza, la conducta- es la base del tratamiento. Por ello, el sistema del juicio (Martin y White, 2005) es central en esta construcción discursiva y opera de manera explícita o aludida en sus dos polos: positivo -la conducta impecable y estricta- y negativo -la conducta adictiva, la recaída o el atracón-. Las opciones verbales que dan cuenta de la conducta que debe adoptar el sujeto refuerzan su lado material, más que mental; son acciones discretas sobre dimensiones espaciales y temporales: "cortar", "medir", "tomar distancia", concordando con la línea médica que propone actuar, más que buscar explicaciones ${ }^{11}$. Ellas conforman una suerte de decálogo moral que definirá la suerte del adicto. La adicción a comer asume en este discurso un rol activo sobre el sujeto, el que debe medirse, controlar una cabeza y un cuerpo que lo desborda. El discurso médico hace ingresar un componente moral a la terapia ("La pregunta que yo siempre me hago es: ¿resulta tan incurable o es tan pobre la voluntad que pone la persona gorda para combatirla?"12), el que transforma al adicto en responsable. La persona es gorda por no tener voluntad, por no pararle "el

10 Idem.

${ }^{11}$ El imperativo es dejar afuera la implicación personal del problema y adoptar el modelo conductual sanador, de una manera acrítica, casi despersonalizada. No se trata de indagar en las causas, las motivaciones más singulares que lo llevaron a la obesidad. El pilar del tratamiento es que los pacientes adopten nuevos hábitos de conducta (Jalif, 2010).

${ }^{12}$ Ver nota 9. 
carro al enanito gordo interno que la hacía resistir indicaciones, no venir cuando tenía que venir, no tomar los minerales, romper y transgredir" 13 .

\subsection{Los saberes reproducidos: la recontextualización del discurso médico}

El discurso médico ingresa y opera como garantía del grupo (Toulmin, 1958). Es invocado explícita e implícitamente, a través de citas textuales, parafraseadas, o por inferencias de conceptos, instrucciones y recomendaciones médicas o psicológicas:

VALERIA: Bienvenida $t u$ acidosis, Luli! (T-E-16/06/16)

PAtricia: Atenti!!! : "El día de hoy termina a las 6 de mañana", Jorge [doctor] dixit. (T-E-19/03/15)

ISABEL: El grupo compartido con Luli me gustó porque entre otras cosas se habló de los desbordes feroces (que suelo tener) y que como Jorge dijo es "cuando el Ello está a pleno, el único que puede pararlo es el Súper Yo. Con el Yo no alcanza"

MARIANA: Lindo que lo compartas Adel, nos hace bien a todas, porque es tal cual lo q dijo Marcelo [doctor]. Más superyo y menos ello... Esto en términos Freudianos... (T-E-21/6/16)

Los conceptos médicos son reproducidos casi literalmente: el descenso es "acidosis", la compulsión es el "ello", el control es "superyó", la persona es "conducta". Estos términos técnicos se insertan en las historias personales ("Bienvenida tu acidosis Luli!"), recontextualizándose en el terreno de lo personal y dando sentido a la vivencia individual de la gordura. El juicio, configurado desde lo discursivo a través del léxico y de lo ideativo, se replica en el plano interaccional, a través de acciones como reportar el peso, monitorear al grupo, comprometerse con la dieta, brindar apoyo en las caídas, celebrar los logros. Estas rutinas, que dan cuerpo a la conducta buscada, se estructuran, generalmente, en tres movimientos:

a) planteamiento del estado de situación (reporte o interrogatorio);

b) invocación o cita del discurso de autoridad; y

c) ratificación grupal del encuadre médico.

En esta secuencia del 31 de marzo (cuadro en la página siguiente), la voz médica es omnipresente. El estilo directo permite reconocer la fuente de esas citas de autoridad, porque son explicitados los nombres de los profesionales. Las secuencias -típicas por lo reiteradas- se inician con un re-

13 Idem. 


\begin{tabular}{|c|c|c|}
\hline $\begin{array}{l}\text { ISABEL } \\
\text { LUCILA } \\
\text { ISABEL }\end{array}$ & $\begin{array}{l}\text { Hola chicas hoy }+500 \text {. No sé por qué } 61.2(58-60) \\
\text { Me voy reportando. Isa, a vos, qué te preocupa de la } \\
\text { oscilación? } \\
\text { Nooo! Nada, no los entiendo demasiado a esos rebotes... } \\
\text { pero bueno venía bajando muy bien los últimos días }\end{array}$ & $\begin{array}{l}\text { Reporte: } \\
\text { problema } \\
\text { con el peso }\end{array}$ \\
\hline ADELA & $\begin{array}{l}\text { Buenos días chicas! Bien por aquí } 57.8 \text { (57/59). Isa, } \\
\text { a seguir construyendo la flaca desde la cabeza y la conduc- } \\
\text { ta y no tanto desde la balanza. De eso, hablamos ayer en } \\
\text { el grupo. No tanto mantener el gordo a raya, sino construir } \\
\text { el flaco, un flaco que aprenda a ser paciente, tolerante, que } \\
\text { no siempre es o se hace lo que uno quiere. }\end{array}$ & $\begin{array}{l}\text { Reproducción } \\
\text { de la voz médica: } \\
\text { construcción del } \\
\text { lugar del adicto }\end{array}$ \\
\hline MARIANA & $\begin{array}{l}\text { Buen día chiquis! qué importante lo que decís...cons- } \\
\text { truir el flaco, de eso se trata el mantenimiento...sigamos } \\
\text { haciendo todo para poder! }\end{array}$ & $\begin{array}{l}\text { Ratificación } \\
\text { del discurso } \\
\text { médico }\end{array}$ \\
\hline LUCILA & Siiiii!!! Construir al flaco!!! & Reafirmación \\
\hline ADELA & $\begin{array}{l}\text { No sólo estar atentas a la comida que nos dispara, que a } \\
\text { esta altura eso ya lo sabemos. Sino, estar atentas a la con- } \\
\text { ducta que nos dispara. Más que tener a raya al gordo hay } \\
\text { que tener a raya a la loca, que el gordo es una consecuen- } \\
\text { cia de conductas. }\end{array}$ & $\begin{array}{l}\text { Reproducción } \\
\text { de la voz médica }\end{array}$ \\
\hline LUCILA & $\begin{array}{l}\text { Jorge [doctor] dijo que muchas veces generamos situa- } \\
\text { ciones para ir a comer. Ese es un buen ejemplo en mi } \\
\text { caso, machacó que es la adicción que busca justificarse. } \\
\text { Para pensarlo }\end{array}$ & $\begin{array}{l}\text { Ratificación } \\
\text { a través } \\
\text { de la evidencia }\end{array}$ \\
\hline FABIANA & $\begin{array}{l}\text { Hola Amores! A mí me quedó resonando la frase } \\
\text { de Gonzalo [doctor] "la vida te queda grande", de } \\
\text { ahogarnos en un vaso de agua x cuestiones cotidianas, y } \\
\text { cuántas veces me voy a comer en vez de resolver la } \\
\text { pequeñez. }\end{array}$ & $\begin{array}{c}\text { personal } \\
(T-E-31 / 03 / 15)\end{array}$ \\
\hline
\end{tabular}

porte, el de Isabel, quien introduce el problema: tiene 500 gramos más que el día anterior, pesa 61.2 y se ha excedido de su rango, que va de 58 a $60 \mathrm{~kg}^{14}$. La modalidad negativa y epistémica ("no sé por qué", "Nooo! Nada, no los entiendo demasiado a esos rebotes") reforzada por la repetición y los alargamientos abre la primera parte del intercambio. En el turno si-

14 Este tipo de secuencia tiene una variante positiva: cuando el reporte indica que se ha descendido de peso o se ha logrado el mantenimiento. En cualquiera de los casos, la voz médica sirve para reforzar justamente el fundamento tanto de la caída como del logro. 
guiente, Lucila explicita la preocupación, el problema ("qué te preocupa de la oscilación?”), que es cerrado con la autojustificación de Isabel, reivindicando una línea de conducta adecuada. A partir de ese encuadre, comienza el intercambio de refuerzos de las restantes integrantes que buscan explicar las posibles razones de la suba de Isabel, a partir de la cita de conceptos ("construir el flaco, de eso se trata el mantenimiento"), sentencias moralizadoras ("un flaco que aprenda a ser paciente, tolerante, que no siempre es o se hace lo que uno quiere"; "es la adicción que busca justificarse"; "la vida te queda grande, en relación a ahogarnos en un vaso con agua x cuestiones cotidianas") e instrucciones ("a seguir construyendo la flaca desde la cabeza y la conducta y no tanto desde la balanza", "No tanto mantener el gordo a raya, sino construir el flaco"; "más que tener a raya al gordo hay que tener a raya a la loca, que el gordo es una consecuencia de conductas"). De manera coral, el grupo realiza diferentes funciones -aconseja, da instrucciones, recrimina- de las que sólo son voceras. La fuente de esos textos es la institución. Los procesos verbales ("hablar", "decir", "destacar", "resonar") hacen ingresar la voz médica ausente en este grupo, que es la autorizada. Estos discursos son recontextualizados en la historia personal, que opera como evidencia en primera persona de la voz profesional ("Ese es un buen ejemplo en mi caso", "cuántas veces me voy a comer en vez de resolver la pequeñez"). Las secuencias se cierran con turnos valorativos, afirmativos, que señalan el alineamiento de las pacientes con los juicios emitidos por los médicos.

En el plano interpersonal, las expresiones de afecto ("chicas", "chiquis", "amores") refuerzan la relación, y las apreciaciones ponen en escena los ideales de salud y belleza ("la flaca", "el gordo") compartidos por el grupo. No obstante, es el sistema del juicio el que subyace y opera como matriz valorativa hegemónica en todo el intercambio. Las apreciaciones, en ese sentido, pierden su naturaleza como tales, para reencuadrarse en este patrón general y totalizante: "la gorda" o "la flaca" son manifestaciones no sólo de patrones estéticos, sino de conductas socialmente adecuadas o inadecuadas. En el ejemplo, "construir la flaca" está asociado no a la "balanza” (o sea al peso en sí mismo), sino con toda una cadena de juicios como "mantener a raya a la loca", a la "conducta", a no "ahogarse en un vaso de agua", a ser "paciente y tolerante", a "resolver la pequeñez".

El cruce de registros refuerza el carácter heterogéneo del interdiscurso: todo viene bien a la hora de orientar, apoyar y -por qué no- recriminar una conducta que puede ser interpretada de manera diferente a la que Isabel planteó. Las respuestas de las compañeras del grupo colocan a Isabel en una posición dudosa: los presupuestos de cada uno de los comentarios llevan a inferencias como que "no es paciente y quiere hacer lo que quiere"; "la conducta (de Isabel) dispara la subida de peso"; "muchas veces se 
generan situaciones para ir a comer"; "se ahoga en un vaso de agua", etc. Como vemos, la responsabilidad recae en el propio paciente, ya que el tratamiento nunca es puesto en duda. En esta estrategia juega un rol central un repertorio de palabras vinculadas con el sistema valorativo del juicio referidas a la culpa y el pecado: la "impecabilidad"15 y sus variantes ("impecable", "impecabilísima”, "impecabilísimo"). El Diccionario de la Real Academia indica que proviene del latín (impeccabilis) y tiene dos acepciones: "exento de tacha" e "incapaz de pecar". Estos adjetivos definen la buena conducta y sus resultados, pero también, su reverso, la frustración y las recaídas.

Fabiana: Hola! Qué bueno acompañarnos! Acá estoy atenta. Con Dieta de descenso. Vamos x la continuidad y la impecabiliidad. EXITO! (T-E03/03/15)

MARIANA: Gracias chicas! Impecabilisima y estrictísima!!!! Qué difícil escribir estas palabras..... Mejor aceptarlas y cumplirlas antes que escribirlas!!Jaja!

AdELA: Buen día bellas mujeres!!! Hoy -800 sigamos impecabilisimas y estrictisimas!!! (T-E-10/03/15)

LuCILA: Cierro mi día, lamento no impecable... Con vergüenza les deseo buen descanso (T-E-02/04/15)

En muy contadas excepciones -apenas tres situaciones en un año y medio de interacción- se cuestiona este encuadre y, en esos casos, la crítica a la autoridad se diluye rápidamente por la reivindicación del discurso médico fuente:

MILI: El primer atracón q tuve fue un día q me pese y a pesar de hacer todo impecable había aumentado 300 gramos, registrar todo me frustró y me llevó a la locura.

MiLI: Si te sirve y no te fomenta la obsesión, podés anotar lo q comes y ajustar detalles (leche, fruta, aceite, verduras)

MILI: Pero si te va a volver más loca, no es preferible que sigas haciendo lo que estás haciendo y en algún momento el cuerpo va a responder?

MiLI: Paz y ciencia?

SILVIA: y sí, paciencia, es que los adictos somos PEGAJOSOS... Nos quedamos pegados a sustancias, personas y lugares.

MARIANA: Jajajaj es asiii Vamosss, almuerzo impecable (T-E-01/06/15).

En esta secuencia, Mili discute el método terapéutico de la institución, basado en el registro constante de los alimentos ${ }^{16}$. La segmentación de la secuencia en diferentes movimientos imprime un ritmo de lectura que re-

\footnotetext{
${ }^{15}$ Estas palabras son las que en el rastreo cuantitativo del corpus más frecuencia de uso tienen.

${ }^{16}$ Puede verse un correlato de ese registro en los reportes del grupo en el whatsapp.
} 
crea la prosodia de una argumentación coloquial (Noblia, 2009). El punto de partida de este cuestionamiento es la evidencia del fracaso del método a pesar de haberlo cumplido rigurosamente ("El primer atracón q tuve fue un día q me pesé y a pesar de hacer todo impecable había aumentado 300 gramos"). La estrategia de Mili es apelar a los conceptos que el discurso médico y el grupo utilizan para definir la conducta positiva ("impecabilidad" "registrar todo"; "anotar lo q comes y ajustar detalles") y recontextualizarlos en resultados que son negativos ("me frustró"; "me llevó a la locura", "te va a volver más loca"). Sobre esos datos se introduce la tesis fuerte de su posición "Pero si te va a volver más loca, no es preferible que sigas haciendo lo que estás haciendo y en algún momento el cuerpo va a responder?”. La modalidad interrogativa opera estratégicamente al dejar en suspenso su grado de certeza, mitigar el argumento, abriendo al grupo la negociación de la conclusión. El argumento de Mili desafía la línea de tratamiento, contraponiendo otra posición que también proviene del campo médico, pero que no es la que sigue el grupo. Esa posición es provocativa porque asocia el tratamiento con la obsesión, otra conducta que también es compulsiva y destructiva. El fin de su intervención se da a través de una valoración "paz y ciencia?" (paciencia), también bajo la modalidad interrogativa.

Es interesante ver cómo el grupo gestiona esa divergencia. La pregunta retórica final abre la posibilidad del grupo para focalizar sobre ese último movimiento y desestimar la argumentación precedente. Contextualizar su intervención en el acto retórico y no en la argumentación, le permite a Silvia eludir el debate y reforzar la posición del grupo desde la valoración y en términos persuasivos. En otras palabras, no importan los argumentos, sí la actitud: Silvia responde indirectamente y ad hominem el cuestionamiento ("los adictos somos PEGAJOSOS... Nos quedamos pegados a sustancias, personas y lugares”). En otros términos, el desafío a la autoridad médica proviene de una adicta (Mili) y, como tal, es un razonamiento enfermo. El cierre del intercambio se da coralmente, a través de la modalidad afirmativa y las risas que apoyan la posición de Silvia y la reivindican del discurso médico que la sostiene, a partir de sus conceptos más caros de esta posición: "impecable" y "sobrio".

El diálogo de las dos posiciones en juego -tanto la que cuestiona el tratamiento (Mili) como la que lo reivindica (Silvia) - las constituye en voceras de dos voces alternativas (Goffman, 1981), que provienen del campo médico y que dan sustento científico al problema. Estos saberes, a su vez, se ven cruzados con otros que provienen del sentido común y las experiencias personales de cada una de las integrantes del grupo, dando lugar a un discurso pastiche (Jalif, 2010) que es poderoso, en la medida en que suma a la voz científica autorizada toda una red de conocimientos diversos que pueden fundamentar mágicamente cualquier argumento. En ese sentido 
se torna incuestionable y "puede defenderse con las piruetas argumentativas más caprichosas", "al no tener lógica clara ni cierta que lo estructure, al ser tan flexible y multiforme, no ofrece puntos de fisura" (Jalif, 2010). Las integrantes del grupo reproducen estas representaciones médicas, las integran a su biografía como marco de interpretación, asumen su decálogo moral y la sufren en cada caída.

En estos casos podemos ver de manera clara el cambio de posicionamiento (Goffman, 1981) de las participantes: de adictas a voceras de la voz de la salud. En esta reproducción, el léxico médico convive con términos de uso cotidiano, del sentido común y de conocimientos de divulgación, los que lo sostienen y refuerzan al reponer, desde otros paradigmas, los mismos núcleos conceptuales. La asunción acrítica y dogmática de las definiciones médicas, por parte de las pacientes, supone un alineamiento doctrinario y también una actitud de subordinación: esa voz autorizada por la ciencia es la fuente de un discurso que el grupo en su totalidad defiende taxativamente ${ }^{17}$ y que las pasiviza al asignarles un rol de ejecutoras de una acción que es instruida por otro. Adoptar ese discurso y reproducirlo sistemáticamente implica asumir un rol subordinado en dos sentidos: como portavoz de registros que les son ajenos, y como paciente, como adictas.

\subsection{El adicto al comer}

Como podemos ver, los contenidos de las categorías centrales del discurso médico sobre la obesidad y los obesos son estructurantes de las identidades de las integrantes del grupo. Hay un complejo metafórico que se pone en juego, constituido por tres metáforas diferentes, pero vinculadas conceptualmente:

a) la adicción y su control como una relación y un proceso espaciotemporal;

b) la adicción y su control como acciones discretas sobre el espaciotiempo: cortar/medir/tomar distancia;

c) la adicción y su control como una escisión cuerpo-mente / cuerpocabeza.

A partir de estos tres ejes, se configuran las identidades personales, grupales y la pertenencia o no al grupo.

\footnotetext{
${ }_{17}$ Pudimos observar, del análisis de los grupos terapéuticos presenciales, que la voz autorizada opera directamente, entablando un contrapunto con la del paciente. En esos casos, la relación es asimétrica y el contrapunto de posiciones pone en escena no sólo roles diferentes sino la asimetría en términos de representaciones.
} 
a) La adicción y su control como una relación y un proceso espaciotemporal. La palabra "adicción” y su familia de términos ("adicto", "adictivo") aparecen de manera explícita, representada ideativamente (Halliday, 2004) a través de procesos y roles que refieren a ella o por alusión:

EugenIA: Chicas hoy en 61,2 -200. Ayer pude pasar la incomodidad de la tarde. Mili vamos juntas. Hoy lo hago x vos. Me voy a reportar $x \mathrm{co}^{-}$ mida. No te quedes en ese lugar, arrancá. Busca alternativas. Para mí el grupo es fundamental. Escucha las palabras amorosas de Ade, Patri y Luli. Todas estamos enfrentando la adicción. Es un largo camino!!!!! (T-E-02/09/15).

SiLviA: Vale querida. Te pasa que no querés largar la comida. La nena caprichosa no quiere dejar esos gustitos que te estás dando. Menos pensar y más hacer. Dieta impecable de descenso es el camino correcto. A la adicción no se la explica, se la expulsa (T-E-02/10/15).

ISABEL: Holaaaa chicas hoy volvi a rebotar, subo, bajo... pero qué sé yo... es lo q me pasa siempre, es la adicción que vuelve (T-E-28/09/15).

La "adicción" en estos textos se plantea como una entidad que trasciende al sujeto, como un enemigo externo que se mueve, que avanza sobre un territorio que es el cuerpo. Los procesos materiales "enfrentar", "expulsar" colocan al sujeto en una compulsa con un actor externo, material, objetivado y sutil, que incluso puede "colarse". Ante este enemigo, el sujeto debe ser irreflexivo pero tenaz ("no se la explica, se la expulsa"; "Menos pensar y más hacer"). La acción material (expulsar) se contrapone a la acción verbal (explicar), en términos de consecuencias: no importa el razonamiento, sino la acción, el imperativo sostenido en las recetas de los profesionales. La colocación (Halliday, 2004) abre otro campo de significaciones espaciales y temporales, que definen una red de metáforas de la adicción y el adicto: "camino", "espacio", "salir adelante”, "subir”, “cerrar”, "bajar”, “caer”, "rebotar”, "volver”, “entrar”, "camino tortuoso", "paso a paso", "comida a comida", "perder la brújula, el mapa”, "compañeras de ruta", "límite”, "caminos que no se cruzan", "autopista”, "paso atrás”, "tierra prometida”, "cerrar el día”, etc.

Adela: Hola Pat. A pensar en el ahora y en mañana. Vamos de nuevo juntas. Paso a paso. No te castigues. Es una adicción. Aunque a veces no parezca, aunque tenga distintas intensidades, aunque se disfrace: es así, es un camino tortuoso (T-E-21/06/16).

MILI: Me deje ganar por la gorda y no la puedo sacar, volvi a caer.

MiLI: Yo soy a todo o nada. Nunca el medio. Nunca el equilibrio, la medida 
Mili: No sé cómo volver. Como si hubiese perdido la brújula, el mapa (T-E26/07/15)

Fabiana: Hola queridas Compañeras de Ruta. Buen dia! 57.9 (período menstrual, mediante), entrando a la autopista, 500, continuidad e impecabilidad... (T-E-30/03/15)

Adela: Ya basta Mili. Un límite. Vos podes decir no. Vos podés ser impecable, cuesta porque la adicción hace lo suyo. Pero podes!! Paso a paso. Comida a comida. No mezcles, son dos caminos que no se cruzan... (T-E-28/08/15)

PATRICIA: Buen día chicas! Hoy 59,2 pero indispuesta (57/59). Estos días de vacaciones estoy haciendo el plan de mantenimiento. No el de descenso (para llegar al piso). Por lo que estuve experimentando llegar al piso parece como llegar a la tierra prometida!!! (T-E-04/03/16)

LUCILA: Cierro mi día, lamento no impecable... un día perdido, un paso atrás, con vergüenza les deseo buen descanso (T-E-02/04/15)

Las metáforas del espacio cruzado por el tiempo representan de manera no congruente particularidades de la adicción: la continuidad del control, la acción siempre en presente o a corto plazo ("Paso a paso. Comida a comida") la responsabilidad del paciente en esa conyuntura ("Cierro mi día, lamento no impecable... un día perdido, un paso atrás, con vergüenza les deseo buen descanso”); la enorme distancia con la meta ("llegar al piso parece como llegar a la tierra prometida").

b) Otra de las metáforas de la adicción y su control aluden a acciones discretas sobre el espacio-tiempo: cortar/medir/tomar distancia, que se expresa verbal o visualmente, a través de emoticones como la tijera, la regla y el avión.

FABIANA: Crees q podes? O necesitas un "refuercito"? Yo estoy en la misma, entrenando impecabiliidad. Nos reportados si querés? Corte, medida y distancia!!!

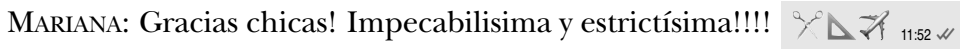

c) La escisión cuerpo-mente/cuerpo-cabeza es un correlato del "mal cerebral", "la falta de voluntad", el problema de conducta tan enfatizado por el discurso médico. Como veíamos anteriormente, la "cabeza" condensa a este sujeto fragmentado, reinstalando la dicotomía occidental mente-cuerpo. La obesidad se desplaza a la "cabeza" de la persona, que es definida como un todo a partir de esa parcialidad. Es una categoría cerrada en la medida en que no admite el ingreso de otras para configurar a un sujeto más complejo.

Adela: 59.300 (57/59). Cabeza adicta. Apretando dientes (T-E-11/04/15)

VALERIA: Más cabeza, menos comida (T-E-18/03/15) 
ADELA: A seguir construyendo la flaca desde la cabeza y la conducta, y no tanto desde la balanza (T-E-31/03/15)

La metáfora del sujeto dividido en términos de cuerpo-mente se replantea en la dicotomía cabeza/dientes, cabeza/comida, recontextualizándola en el campo semántico del comer. En algunos casos, el tópico que determina la práctica opera por síntesis: la cabeza es "flaca" o "gorda", asimilando en ese proceso al cuerpo y a la conducta:

MARIANA: Chiquis por acá todo bien...contenta porque mis compañeras de viaje son deportistas y flacas de cabeza (T-E-29/5/15)

Patricia: Estoy llena. Es de la cabeza. Lo sé, lo sé!! (T-E-24/05/15)

AdELA: (...) los kilos de la cabeza son los que más nos pesan (T-E-02/09/15)

Esta síntesis la vincula a esta adicción con otro problema mental, la locura. La cabeza pasa a ser la sede de pensamientos erróneos, puede funcionar mal e, incluso, perderse o intercambiarse si no funciona correctamente.

Lucila: Buen día! 58,400 (58/60) Gracias, Pat x ayudarme en mi locurita vespertina $\mathrm{x}$ ayer!!!!!

PATRICIA: Cene muy bien pero tengo un poco de inquietud poco sana!! Es la primera vez que aviso antes.., no sé que es. A la tarde me fui a dormir la siesta porque había dormido 4 hs a la noche pero también para irme de estos pensamientos (T-E-16/04/15)

MARIANA: Exacto, distancia es la única forma, y mucho líquido...la $\mathrm{ca}$ beza/en automático con este tema (T-E-26/03/15)

LuciLA: Merienda y cena bien pero no impecable... See quedó en alguna caja de la mudanza la cabeza prestada... Aquí la tomo de nuevo, cierro el día (T-E-27/06/15)

MARIANA: Chicas cena perfecta cierro acá contenta de/retomar la sen$\mathrm{da}$, sigamos con la cabeza que nos prestamos cuando no tenemos la propia, vamos que juntas podemos! (T-E-27/06/15)

El entretejido conceptual es poderoso, porque es redundante sin ser repetitivo. Los conceptos se van entrelazando saturándose: la adicción es conceptualizada como espacio (camino), como movimiento (paso a paso), que a su vez se vincula con el tiempo (día a día) y los alimentos (comida a comida), que a su vez se relacionan con acciones (cortar, medir y tomar distancia). Como podemos ver, el cruce de estas metáforas va configurando, en un mismo movimiento, una representación compleja de la adicción a la comida, de la obesidad y del obeso. Sirve para dar cuenta de los procesos de mantenimiento exitosos y de los frustrados, según sea con una mo- 
dalidad, una actitud y una gradualidad positiva o negativa (Halliday, 2004; Martin y White, 2005). Los distintos sistemas vinculados al juicio, los metafóricos o los explícitos, se suman y dan lugar a una representación compleja de la enfermedad.

AdELA: Hola Chicas. Tuve una recaída. Lamento dar estas noticias pero sinceridad ante todo. Fue fuerte, venía peligrosa con la cabeza por eso fui al grupo pero aún asi caí. A levantarse y a seguir. Enfermedad recidivante...

MARIANA: Ade, coincido con vos...el estar tan comprometidas con nuestro deseo de delgadez y sobriedad y con nuestro grupo hace que si tropezamos enseguida nos levantemos...y de eso se trata...hacer todo lo que hay que hacer para no tropezar... pero si sucede, enseguida levantarnos y volver a ser impecables! $\therefore$ (T-E-02/05/15)

LucILA: Buen día! 58,70 = ayer (58/60). De la cabeza MAL. Derrapé muyyyy mal Comi inadecuado a 1/2 mñn... Hoy corte, medida y distancia. Me reportaré c/comida y tendré agua EN la mano el resto de la jornada, será separar los caminos... (T-E-21/06/15)

PATRICIA: Hola chicas!! Patiné re mal. Venía re bien y estando indispuesta todos los días pesaba más. No se. Se me quemó la cabeza. Me frustré y aflojé. No comí nada raro pero saqué atención a las porciones. Ahora perdi el terreno ganado. Ahora mismo estoy con la idea de hacer lo q se q no está bueno. Por eso lo escribo (T-E-21/06/16)

La culpa y la vergüenza, si bien son rechazadas desde el discurso médico oficial, son fomentadas por sus representaciones. La falta de voluntad, de conducta, el no poder controlar la adicción es visto como una falla de la persona y es condenada. La metáfora espacial en sus diferentes manifestaciones ("camino", "caer", "levantarse", "tropezar”, "derrapar”, "alejarse”, "perder territorio", "retroceder”, "patinar", etc.) se combina con la metáfora de la cabeza ("Cabeza un poquito inquieta", "Se me quemó la cabeza"; "De la cabeza MAL") y la del corte ("Hoy corte, medida y distancia"). A ellas se suma la red de juicios explícitos ("volver a ser impecables"; "Todo impecable”), generando un discurso cerrado, circular. El registro da cuenta de la adicción no sólo porque la representa sino también porque se satura circularmente. Una metáfora se representa en término de otra y cualquiera de ellas puede operar por sustitución de la otra. Todas ellas son tan homogéneas y poderosas que le quitan peso a la individualidad. No hay estilos personales, apenas marcas dialectales que el whatsapp pone en escena gracias a la oralidad que ingresa a través de los audios. La representación individual de cada integrante se reduce a la membresía del grupo. El grupo las representa y viceversa. 
ADELA: Ya sabemos qué podemos comer y qué no. Hagámoslo! Necesito cortar y para eso las necesito a ustedes bien. Nos ayudamos? Hacemos un día perfecto hoy? Si alguna come, engorda el grupo.... no solo en la balanza, los kilos de la cabeza son los que más nos pesan (T-E-02/09/15)

Dada esta identificación tan homogénea, el ingreso al grupo no es automático: este rito de pasaje debe garantizar que el ingresante comparta el propósito, las reglas, los valores y las representaciones del grupo:

Fabiana: Hola! Buen dia! Ayer Patricia (la chica q hizo el cruce de los Andes y q la tuvieron q operar xq se fracturó, me dijo q quería participar de un grupo de wapp, si les parece bien, la sumamos. Parece q es de cuidarse mucho habitualmente y ahora le está costando. ..

LuCILA: Si la ubico, creo, siempre es bueno un aporte si está comprometida no?

MARIANA: Chicas la conozco bien, corre en el team del novio de Laura, la q me trajo al Centro... es la chica q/habló ayer al final morocha, q el doctor le habló bastante... Creo le va a hacer bien el contacto diario!! Ree comprometida estuvo y está siempre...creo nos va a aportar...

[Adela ha añadido a Patricia] (T-E-07/04/15)

En ese sentido operan, también, las tres rutinas básicas que estructuran la interacción:

a) las de reporte, a través de las cuales las participantes informan a las demás de su peso y su rango (el piso y el techo del peso que los médicos les han definido):

MARIANA: Buen día chiquis! Hoy 52,100 +100 q ayer, (51/53) a correr y a bailar... Lucila: Buen día! 59,30 -200 (58/60) hoy consulta con Sofy y creo q almuerzo en el Centro

ISABEL: Holaaaa chicas buen día!!!! -600 63 (58/60)

VALERIA: Buen día reinas!! Hoy 61,2 (57/59) -400 Quiero salir del 61 y llegar a mi franja!!! Convicción en mi deseo! Vamosssssssss!!! (T-E$06 / 03 / 15)$

b) las de control, que tiene que ver con confirmar el cumplimiento de la rutina y el ajuste de las representaciones:

ADELA: Luli, cómo va? Mari? Mili?

MiLI: Yo cerré el día pero no impecable. Mañana será otro día renovando el compromiso 
MARIANA: Yo bienn chiquis hay pollo así q comeré mi porción...vengo perfecta! Gracias por estar!! (T-E-19/03/15)

ISABEL: El peso de hoy es 64.1 y mi banda es 58/60

ADELA: Repórtate Isa, comida a comida hasta que pasen 3 o 4 días. Vas a hacer dieta de descenso?

ISABEL: Siiiiii las necesitoooov gracias

ADELA: Buena decisión! En ácidosis es más fácil pero tenes que ir paso a paso, comida a comida.

ISABEL: Siiii ya lo sé, súper despacio

Adela: No se trata de despacio o rápido. Se trata de ir pudiendo, sabes? Paso a paso se van completando los días continuos de dieta impecable.

ISABEL: Ya lo seee.... quise escribir de a poco...

ADELA: Entonces escribi “de a poco" (T-E-02/03/15)

c) las de apoyo y contención, que son puramente fáticas y que implican celebrar los logros y reorientar y sostener las recaídas:

ADELA: Si alguna anda mal: cabeza prestada. Y paso a paso. Juntas podemos $(T-E-31 / 7 / 15)$

Mariana: Mili, cabeza prestada. No te escuches, tu cabeza está tomada. Te prestamos nuestra cabeza (con algunas arrugas ji), hacenos caso. Vas a salir $(T-E-28 / 8 / 15)$

La pertenencia y la exclusión del grupo se vinculan directamente con el contenido que se le atribuye al trastorno alimentario. Según la perspectiva médica se plantean en el grupo, al menos, dos posibilidades: encuadrar la categoría obesidad como adicción u obesidad como "enfermedad". Estas opciones, no obstante, no surgen del grupo: una vez más se imponen desde afuera, desde la autoridad del discurso médico. Cuando estos dos marcos entran en colisión, el grupo entra en crisis. Una de las situaciones se plantea cuando Mili les avisa a sus compañeras que va a dejar el grupo porque en vez de mejorar el tratamiento la fue llevando -según sus palabras- a enfermarse cada vez más. La dieta prescripta por los médicos la condujo a una seguidilla de restricciones y atracones que, en una consulta a otro centro, diagnosticaron como bulimia nerviosa. El texto de despedida es oral y con una profunda carga emotiva. El mensaje de voz ingresa a las 12:30. La secuencia en la que se inserta este texto es la siguiente:

12:30:01: Mili: (nota de voz)

12:34:02: ADELA: Hola Chicas! Hola Mari!

12:35:16: AdElA: Escuche tu audio y lo primero que vino a mi cabeza fue

"Mari está en la autopista!"!!! Me alegro mucho y te felicito.

12:37:10: Adela: Yo ando intentando subir, y lo voy a lograr. Ayer fui al grupo de la 19 hs y me hizo bien. Hoy voy de nuevo y mañana también. Besos a todas. 
12:37:44: ADELA: Si pueden y quieren cuenten como andan.

12:41:14: Patricia: Yo muy bien. -200. 60k (57/59) bajando poquito pero continuo

12:54:21: MARIANA: Gracias chiquis!! Me hacen muy bien sus palabras para seguir en la autopista!

Continuo.. sin pausa con objetivo claro! Vamos Adeli, juntas por la autopista es más lindo (...)

15:09:05: Mili abandona el grupo (T-E-16/10/15)

A pesar de estar activa, la conversación grupo ignora el mensaje afectuoso y emotivo de Mili y prefiere responder a un audio muy optimista que Mariana había enviado el día anterior, en el que contaba los logros con el tratamiento. La falta de respuesta del grupo es de rechazo explícito, al punto que luego de esperar casi tres horas, Mili abandona el grupo sin recibir ningún mensaje. Una vez sucedido esto, comienzan a conversar sobre el tema. La secuencia se inicia con Adela, que hace referencia a la situación:

15:27:27: AdELA: El audio de Mili fue muy fuerte para mí porque me hizo acordar a mi adolescencia y unos años más también. Su angustia es mucha. Deseo de todo corazón que le vaya bien en el nuevo centro al que está yendo acompañada por sus padres. Yo pasé por varios lugares especializados en bulimia, pero lo único que me ayudó finalmente fue madurar un poco, terapia individual y venir al Centro.

Todas son conscientes de que Mili ya no está en el grupo, de ahí su referencia en tercera persona y no en segunda, como era usual. Los argumentos de Adela son corroborados por el grupo, que se hace militante de la representación médica de la obesidad como adicción. Incluso cuestionan el nuevo encuadre del problema ("Yo pasé por varios lugares especializados en bulimia, pero lo único que me ayudó finalmente fue madurar un poco, terapia individual y venir al Centro"). La construcción de la propia patología se ha encuadrado dogmática y acríticamente en la definición que el Centro al que asisten le asigna al problema y no se pone en cuestión, ni siquiera cuando ésta parece no dar una respuesta a las recaídas o al tratamiento sin resultados. El encuadre de la obesidad como adicción parece brindarle a la autoridad médica y a las mismas pacientes la justificación indiscutible y circular tanto del éxito como de su fracaso.

\section{Conclusiones}

A modo de síntesis, podemos hacer una sistematización del análisis realizado: 
- Alta ritualización: los patrones conversacionales se reiteran casi sin variación, generando una estructura cíclica asentada en las rutinas de reporte, control y apoyo. Si bien hay algunos roles definidos por el monitoreo y el control del grupo (Adela y Mariana), en general los patrones interactivos (los microrrelatos, las afirmaciones y evaluaciones, las secuencias de preguntas y respuestas) son ejercidos por todas y están pautados por la meta y el tópico.

- Estilo uniforme: el registro del grupo se impone unificando las intervenciones. Las voces individuales se pierden en un colectivo coral que reproduce casi mecánicamente la voz autorizada. El léxico está acotado a un repertorio de palabras y metáforas asociadas con el tópico general de la obesidad, que cruza terminología técnica, con expresiones coloquiales y de sentido común. El registro médico marcado por las impersonalizaciones, nominalizaciones y la asimetría que instituye la voz profesional se conjuga con otro definido por la subjetividad del yo, propio del testimonio, de la historia personal y de la simetría de una conversación entre partes. El choque entre un discurso que se aleja del sujeto para plantear el tema en término de procesos, relaciones y abstracciones, con otro anclado en la vivencia individual, da lugar a un pastiche que permite justificar las recaídas en términos de la propia adicción.

- Multimodalidad: la escritura y la oralidad operan complementariamente, aunque prima la escritura para las rutinas ordinarias. La oralidad aparece cuando el contexto restringe la posibilidad de escribir o cuando sucede algún acontecimiento excepcional (muerte del hermano; casamiento de un exmarido; despedida del grupo), que requiere extenderse en el tiempo y emotividad. El control constante, la ubicuidad de la interacción, la inmediatez de la respuesta, propios de los usos del whatsapp, refuerzan las posibilidades de registrar y supervisar la vida de cada integrante. La tecnología facilita el apoyo tanto como el disciplinamiento; a la vez que -paradójicamente- hace omnipresente el objeto de la adicción, la comida. Lejos de distanciarlas del problema, las sumerge de manera compulsiva en esa realidad.

- Las representaciones sociales: el repertorio léxico y metafórico que se utiliza para referir a la obesidad es restringido. Se organiza a partir de tres dimensiones semánticas espaciales y temporales, que dan cuenta de un núcleo conceptual básico que es la adicción: localizada en la cabeza/conducta; como un proceso siempre presente y continuo ("camino", "paso a paso"); y controlada a partir de tres acciones básicas y discretas que definen su tratamiento: "cortar", "medir" y "tomar distancia". Este repertorio discursivo y la prác- 
tica misma identifican a las integrantes de un grupo que, fuera de este espacio, no es homogéneo en términos sociales, económicos y etarios.

Las representaciones del grupo las muestra como personas siempre fuera del eje o en peligro de salirse: "la gorda", "la loca", "la flaca" refieren a los polos del exceso. El término medio, "la normalidad", no define lo deseable, que también es un estado fuera del parámetro (la delgadez). El desplazamiento de los atributos de la enfermedad a la persona (de la "obesidad es una adicción" al "somos adictas"), por otro lado, fija el estigma en la persona, que está condenada de por vida al tratamiento, a la vez que -paradójicamente- funciona como un refugio, en la medida en que le permite justificar los fracasos. La concordancia entre las representaciones médicas y las de las pacientes, forma un complejo discursivo poderoso y homogéneo que resulta incuestionable y opera como un marco de producción e interpretación de una comunidad de prácticas que no admite desvíos en el foco de la interacción.

En este registro, la modalidad asertiva rara vez se quiebra, mucho menos cuando se cita la voz médica. Es un tono afirmativo que no admite matices y que refuerza el sistema de juicio. Este discurso resulta, por momentos, monoglósico, en el sentido en que no permite el ingreso de un contradiscurso que refute la validez médica del discurso dominante, el del centro de salud que las encuadra. Los pocos casos en los que éste y su efectividad son puestos en duda, la estrategia pasa por desplazar la responsabilidad a la primera persona, a la paciente, ratificando la voz médica. La dimensión moral del discurso médico, que atribuye un rol fundamental a la voluntad del paciente, se reproduce en el grupo no sólo a través del sistema semántico-discursivo del juicio, sino también en su dimensión interaccional: puede relevarse en la configuración de identidades, posiciones y valores de los participantes, pero también en las acciones que el grupo lleva a cabo a través de sus rutinas de control. Éstas conllevan una carga valorativa, al operar sobre la conducta considerada positiva o negativa. Los interrogatorios, los reportes y testimonios que las mismas participantes realizan día a día y durante diferentes momentos del día ponen en evidencia el alto grado de disciplinamiento del grupo a una práctica social signada por la vigilancia. En este sentido, el grupo de whatsapp forma parte de los distintos dispositivos con los que cuenta el centro de salud para el control de sus pacientes (la clínica, los grupos, los gimnasios y restaurantes, las viandas, etc. ${ }^{18}$. Las

\footnotetext{
${ }^{18}$ Si bien estos grupos operan fuera del circuito institucional y se dan entre pares, los supuestos, grados de conocimiento y expectativas están asociados al marco institucional que las convoca. En ese sentido, las identidades replican los mecanismos de autoridad y control,
} 
conductas "impecables" son celebradas, las no "impecables" son perdonadas, en la medida en que sean acompañadas por un acto de contricción y de reivindicación de los valores y el compromiso instruidos. Este esquema es circular y da cuenta de la dimensión adictiva de la enfermedad, a la que el discurso médico responde en sus mismos términos: el tratamiento genera la misma dependencia y circularidad que la enfermedad a la que trata.

El condicionamiento de la representación social impuesta por el discurso médico es poderoso y justifica la circularidad del comportamiento adicto. El contraste entre concebir la obesidad como adicción o concebirla como enfermedad es un límite difuso pero significativo. En este grupo no hay enfermos (salvo por gripe o ciática) sino adictos. Transformar una adicción indefinida, por una patología tipificada, por ejemplo, la bulimia, significa un cambio de marco fundamental en término de relaciones y conductas: el grupo no puede contener a un enfermo, aunque los síntomas sean los mismos. De ahí que la autoexclusión sea una de las consecuencias del peso de esta clasificación. Ser tipificado como "enfermo" supone otra identidad y, por ende, otro grupo. Enmarcar la misma patología desde marcos diferentes transforma a la persona, su identidad y sus posibilidades de vida. En este marco, la gordura concebida en términos de enfermedad supone una temporalidad que puede cerrarse por la aplicación de diferentes tratamientos (quirúrgicos, químicos y psicológicos), un proceso que en definitiva puede tener un pasado, un presente y un futuro cerrado. En contraste, la adicción supone un presente continuo e imperfecto. Las metáforas temporales y espaciales son estructurales: hay un piso y un techo, y en el medio un espacio indefinido marcado por un continuo reportarse y restringirse; un presente continuo determinado espacial y actitudinalmente: un camino, un paso a paso que lleva a ese estado ideal siempre imperfecto, siempre inestable, siempre promisorio y, por ende, siempre futuro. La reincidencia como rasgo típico de la adicción no augura un futuro que no sea de control y de recaídas. En un año y medio de intercambios, nada, ni el compromiso más grande, ni la dieta más estricta, ni el apoyo de profesionales y grupos de pacientes, logra cortar el círculo que las ata a la comida y al tratamiento de una manera patológica.

la ideología y los sistemas de creencias que les da la institución médica. Es en ese sentido un diálogo institucionalizado (Drew y Sorjonen, 1998), que asumen de forma vicaria y reproducen con su identidad, sus acciones y rutinas en cada intercambio cotidiano. 


\section{BIBLIOGRAFÍA}

Cormillot, A. (2008): Cómo adelgazar y mantenerse con el Dr. Cormillot, Buenos Aires, Paidós.

FAirclough, N. (2003): Analysing Discourse, London, Routledge.

Halliday, M. (2004): An Introduction to Functional Grammar, London, Arnold.

Goffman, E. (1974): Frame Analysis. An essay on the organization of experience, Boston, Northeastern University Press.

Goffman, E. (1981): Forms of talk, Philadelphia, Philadelphia University Press.

GUBA, E. G. y LinCOLN, Y. S. (1994): "Competing paradigms in Qualitative Research”, en N. K. Denzin e Y. S. Lincoln, Handbook of Qualitative Research, California, Sage.

JALIF, K. J. (2010): El dispositivo grupal en el tratamiento de la obesidad (Tesis), Buenos Aires, Universidad de Belgrano.

Kress, G. (2010): Multimodality: A Social Semiotic Approach to Contemporary Communication, London, Routledge.

Martin, J. R. y White, P. (2005): The Language of Evaluation. Appraisal in English, New York, Palgrave Macmillan.

Noblia, M. V. (2009): "Modalidad, evaluación e identidad en el chat", Discurso y Sociedad, 3 (4), págs. 738-768.

Noblia, M. V. (2012): La relación interpersonal en el chat. Procesos de construcción y negociación de la identidad, Buenos Aires, Universidad de Buenos Aires.

Noblia, M. V. (2015): "De la alternancia de códigos a la alternancia de modos: las nuevas modalidades del argot en las tecnologías móviles", en O. Conde (ed.), Argots hispánicos. Analogías y diferencias en las hablas populares iberoamericanas, Lanús, Universidad Nacional de Lanús, págs. 273-297.

Organización Mundial de la SAlud (2016): "Obesidad y sobrepeso", Nota descriptiva, 311, Junio, accesible en: <http://www.who.int/mediacentre/fact sheets/fs311/es/>. Visitado el 23/04/2016.

SACKs, H. (1992): Lectures on Conversation, Oxford, Basil Blackwell.

Stommel, W. (2009): Entering an online support group on eating disorders. A discourse analysis, Amsterdam, Rodopi.

Toulmin, S. (1958): The Uses of Argument, Cambridge, Cambridge University Press.

Wenger, E. (1998): Communities of Practice: Learning, Meaning, and Identity, Cambridge, Cambridge University Press.

Wenger, E., Mc. Dermott, R. y Snyder, W. (2002): Cultivating Communities of Practice: A guide to managing knowledge, Boston, MA, Harvard Business School Press. 